\title{
Numerical approximation of the maximal solutions for a class of degenerate Hamilton-Jacobi equations*
}

\author{
Fabio Camilli \\ Dipartimento di Matematica \\ Università di Torino \\ Via Carlo Alberto 10 \\ 10123 Torino, Italy \\ camilli@dm.unito.it
}

\author{
Lars Grüne \\ Fachbereich Mathematik \\ Johann Wolfgang Goethe-Universität \\ Postfach 111932 \\ 60054 Frankfurt a. M., Germany \\ gruene@math . uni-frankfurt.de
}

\begin{abstract}
In this paper we study an approximation scheme for a class of Hamilton-Jacobi problems for which uniqueness of the viscosity solution does not hold. This class includes the Eikonal equation arising in the Shape from Shading problem. We show that, if an appropriate stability condition is satisfied, the scheme converges to the maximal viscosity solution of the problem. Furthermore we give an estimate for the discretization error.
\end{abstract}

Keywords: Singular Hamilton-Jacobi equations, maximal solution, regularization, numerical approximation, discretization error

AMS Classification: 65M12, 65M15, 49L25

\section{Introduction}

Given a Hamilton-Jacobi equation, a general result due to Barles-Souganidis [3] says that any "reasonable" approximation scheme (based f.e. on finite differences, finite elements, finite volumes, discretization of characteristics, etc.) converges to the viscosity solution of the equation. Besides some simple properties that the approximation scheme has to satisfy, it is only requested that the equation satisfies a comparison theorem for discontinuous solutions, which in particular implies uniqueness of the viscosity solution.

This result covers a wide class of first and second order Hamilton-Jacobi equations, yet there are interesting examples of equations coming from the applications for which uniqueness of the viscosity solution does not hold. A significant example is given by the Eikonal equation

$$
|D u|=f(x)
$$

on some open and bounded domain $\Omega \subset \mathbb{R}^{n}$ coupled for example with a Dirichlet boundary condition on $\partial \Omega$. This equation arises in the Shape-from-Shading problem in image analysis and a large literature has been devoted to its study (see [4] for a description of the problem

\footnotetext{
${ }^{*}$ This paper was written while the second author was visiting the Dipartimento di Matematica, Università di Roma "La Sapienza" supported by DFG-Grant GR1569/2-1. The research was partially supported by the TMR Network "Viscosity solutions and their applications".
} 
and [16] for a viscosity solution approach). It is well known that if $f$ vanishes at some points, there are infinite many viscosity solutions to (1.1) (see [15]). Nevertheless, among these solutions, in general only one is the relevant solution (for example, from the physical point of view, from the control theoretic one, etc.).

In [6] (see also [14]), requiring a stronger condition for supersolution than that for the standard viscosity solution, a Comparison Principle, which characterizes the maximal viscosity solution of the problem, has been obtained for the following class of Hamilton-Jacobi problems

$$
\begin{array}{r}
H(x, D u)=f(x) \quad x \in \Omega, \\
u(x)=g(x) \quad x \in \partial \Omega .
\end{array}
$$

Here $\Omega$ is a bounded domain of $\mathbb{R}^{N}, H$ and $f$ are nonnegative continuous functions and $f$ can have a very general zero set (the Eikonal equation (1.1) fits into this class of equation). It is worth noting that this maximal solution is the value function of a control problem associated in a suitable way to (1.2)-(1.3).

There are, in general, two approaches to the discretization of problem (1.2)-(1.3).

A first possibility is to discretize problem (1.2)-(1.3) directly, but imposing some additional condition which among the infinite many solutions singles out the one we want to approximate: for example, in [17], it is assumed that the solution is known on the zero set of $f$, which is now a part of the boundary of the domain where the problem is discretized.

A second possible approach (see [4], [5] and references therein) is to discretize a regularized version of problem (1.2)-(1.3), obtained by cutting from below $f$ at some positive level $\epsilon>0$ (note that for $f>0$ problem (1.2)-(1.3) has a unique viscosity solution). To prove the convergence of the scheme, both $\epsilon$ and the discretization step $h$ have to be send to 0 . Since the limit problem does not have a unique viscosity solution, it is not possible to apply the Barles-Souganidis theorem and, to our knowledge, there is no convergence theorem for this class of schemes, at least for a general zero set of $f$. Furthermore, if $\epsilon$ and $h$ are not related by some condition, the approximation scheme shows numerical instability and it is not really known which solution is approximated (see [12] for some numerical tests in this sense).

Aim of this paper is to describe an approximation scheme for which it is possible to prove the convergence to the maximal solution of problem (1.2)-(1.3), without requiring any additional assumptions.

The scheme is based on a two step discretization of the control problem associated to the regularized problem: first in the time variable, discretization step $h$, and then in the space variable, discretization step $k$ (see [2], [13] for related ideas).

In the first part (Sections 3,4), we study the approximation scheme obtained by discretization in time. We show that, if $\epsilon$ and $h$ are related in an appropriate way, the scheme converges to the maximal solution of (1.2)-(1.3) for $\epsilon$ and $h$ going to zero. This result is in the spirit of [3], in the sense that it is based on stability properties of the maximal viscosity solution and on its characterization given by the comparison theorem in [6]. Therefore, the proof of the convergence theorem can be easily modified to manage other boundary conditions instead of (1.3) or, also, different approximation schemes not necessarely based on the control theoretic interpretation of the problem. 
In the second part (Section 5) we study the discretization error for the fully discrete scheme. We show that, if the zero set of $f$ is not too "wild", it is possible to estimate in terms of $\epsilon$ and of the discretization steps the $L^{\infty}$-distance between the approximate solution and the maximal solution of the continuous problem. This part deeply employs the control theoretic interpretation both of the discrete problem and of the continuous one.

\section{Continuous problem: assumptions and results}

In this section we briefly recall the characterization of the maximal solution of problem (1.2)-(1.3) obtained in [6]. Here and in the remainder of the paper by (sub, super)solutions we mean Crandall-Lions viscosity (sub, super)solutions (see [1] for a general treatment).

We first set the assumptions on the data of the problem. The hamiltonian $H: \bar{\Omega} \times \mathbb{R}^{N} \rightarrow \mathbb{R}$ is assumed to be continuous in both variables and to verify

$$
\begin{gathered}
H(x, 0)=0, \quad H(x, p)>0 \quad \text { if }|p| \neq 0, \\
\lim _{|p| \rightarrow+\infty} H(x, p)=+\infty \quad \text { uniformly for } x \in \bar{\Omega}, \\
t \rightarrow H(x, t p) \text { is strictly increasing for } t \in[0,1] \\
\text { for any }(x, p) \in \bar{\Omega} \times \mathbb{R}^{N},
\end{gathered}
$$

and

$$
\mathcal{Z}(x):=\left\{p \in \mathbb{R}^{N}: H(x, p) \leq f(x)\right\}
$$

is convex for any $x \in \bar{\Omega}$.

Note that the hypothesis (2.2) replaces the stronger one of convexity of $H$ in $p$.

The function $f: \Omega \rightarrow \mathbb{R}$ is nonnegative, continuous in $\bar{\Omega}$. Moreover, defined $K:=\{x \in$ $\bar{\Omega}: f(x)=0\}$, it is assumed that

$$
K \cap \partial \Omega=\emptyset .
$$

Finally we assume $g: \mathbb{R}^{N} \rightarrow \mathbb{R}$ to be a continuous and bounded function.

We introduce the gauge function $\rho$ and the support function $\delta$ of the convex set $\mathcal{Z}(x)$, namely

$$
\begin{aligned}
\rho(x, p) & =\inf \left\{\lambda>0: \frac{p}{\lambda} \in \mathcal{Z}(x)\right\} \\
\delta(x, p) & =\sup \{p q: q \in \mathcal{Z}(x)\},
\end{aligned}
$$

for any $(x, p) \in \Omega \times \mathbb{R}^{N}$. Both these functions are convex and homogeneous in the variable $p$, and are 1.s.c. and respectively continuous in $\Omega$ (note that, if $x \in K, \rho(x, 0)=0$ and $\rho(x, p)=+\infty$ for $|p| \neq 0)$. Moreover they are related by the following equality

$$
\rho(x, p)=\sup _{\delta(x, q) \leq 1}\{p q\} \quad x \in \Omega, p \in \mathbb{R}^{N} .
$$

Example 2.1 Let $\psi: \mathbb{R}^{+} \rightarrow \mathbb{R}^{+}$be a continuous function such that $\psi(0)=0$ and $\psi$ is strictly increasing. Consider the equation

$$
\psi(|D u(x)|)=f(x) \quad x \in \Omega .
$$


In this case we have

$$
\begin{gathered}
\mathcal{Z}(x)=B\left(0, \psi^{-1}(f(x))\right. \\
\rho(x, p)=\frac{|p|}{\psi^{-1}(f(x))}, \quad \delta(x, p)=\psi^{-1}(f(x))|p| .
\end{gathered}
$$

We now define a nonsymmetric semidistance on $\bar{\Omega} \times \bar{\Omega}$ by

$$
\begin{array}{r}
L(x, y)=\inf \left\{\int_{0}^{T} \delta(\xi(t),-\dot{\xi}(t)) d t: T>0, \xi(t) \in W^{1, \infty}([0, T], \bar{\Omega})\right. \\
\text { s.t. } \xi(0)=x, \xi(T)=y\}
\end{array}
$$

and, for $x \in \Omega$ and $r>0$, the open sets

$$
B_{L}(x, r):=\{y \in \Omega: L(x, y)<r\} .
$$

It can be shown that the family $B_{L}(x, r)$ induces a topology $\tau_{L}$ on $\bar{\Omega}$. If $K$ consists of isolated points this topology is equivalent to the Euclidean topology and the problem can be studied in the framework of viscosity solution theory (see [14]). In general, $\tau_{L}$ is weaker than the Euclidean topology and, for $x \in K$, the set of points having zero $L$-distance from $x$ is a subset of $K$.

To obtain the characterization of the maximal solution, the definition of viscosity solution will be adapted to the topology $\tau_{L}$.

Definition 2.2 Given a l.s.c. function $v: \Omega \rightarrow \mathbb{R}$, a Lipschitz continuous function $\phi$ is called $L$-subtangent to $v$ at $x_{0} \in \Omega$ if, for some $\epsilon>0$,

$$
\begin{aligned}
& \phi\left(x_{0}\right)=v\left(x_{0}\right) \\
& \phi(x) \leq v(x) \quad x \in B_{L}\left(x_{0}, \epsilon\right) .
\end{aligned}
$$

The $L$-subtangent is called strict if $\phi(x)<v(x)$ outside $B_{L}\left(x_{0}\right)=\left\{x \in \Omega: L\left(x_{0}, x\right)=0\right\}$.

We remark that the convexity assumption (2.2) allows us to use Lipschitz continuous test functions instead of $C^{1}$ test functions as in the standard definition of viscosity solution. For a Lipschitz continuous function $\phi$, we denote by $\partial \phi(x)$ the generalized gradient of $\phi$ at $x$, i.e.

$$
\begin{aligned}
\partial \phi(x)=\overline{\operatorname{co}}\left\{p \in \mathbb{R}^{N}: \quad\right. & p=\lim _{n} D \phi\left(x_{n}\right) \text { for a sequence } x_{n} \rightarrow x \text { s.t. } \\
& \left.\phi \text { is differentiable at } x_{n}\right\} .
\end{aligned}
$$

Definition 2.3 A l.s.c. function $v: \Omega \rightarrow \mathbb{R}$ is said to be a singular supersolution of (1.2) if for any $x_{0} \in \Omega$ and for any $\phi, L$-subtangent to $v$ at $x_{0}$ such that $\partial \phi(x)=\{0\}$ in $x \in B_{L}\left(x_{0}, \epsilon\right) \cap K$, there exists a sequence $x_{n} \in \Omega \backslash K$ and a sequence $p_{n} \in \partial \phi\left(x_{n}\right)$ for which

$$
\lim _{n \rightarrow+\infty} L\left(x_{0}, x_{n}\right)=0
$$

and

$$
\lim _{n \rightarrow \infty} \rho\left(x_{n}, p_{n}\right)-1 \geq 0
$$


It is worth noting that the definition of singular supersolution reduces to the standard definition of viscosity supersolution if $x_{0} \in \Omega \backslash K$. In fact, in this case, since the topology $\tau_{L}$ and the Euclidean topology are equivalent in neighborhood of $x_{0}, L$-subtangents at $x_{0}$ coincide with standard subtangents. Moreover, in $(\Omega \backslash K) \times \mathbb{R}^{N}, \rho(x, p) \leq 1$ (resp. $\rho(x, p) \geq 1)$ if and only if $H(x, p) \leq f(x)$ (resp. $H(x, p) \geq f(x)$ ).

In the following theorem, we compare viscosity subsolutions and singular supersolutions of equation (1.2).

Theorem 2.4 Let $u \in U S C(\bar{\Omega}), v \in L S C(\bar{\Omega})$ be a viscosity subsolution and a singular supersolution of equation (1.2), respectively, such that $u \leq v$ on $\partial \Omega$. Then

$$
u \leq v \quad \text { in } \bar{\Omega} .
$$

Hypothesis (2.2) allows us to give a control theoretic interpretation of problem (1.2)-(1.3). Let $U$ be the value function of the control problem with dynamics

$$
\left\{\begin{array}{l}
\dot{\xi}(t)=q(t) \quad t \in[0, \infty) \\
\xi(0)=x,
\end{array}\right.
$$

where $x \in \Omega$ and $q$ is any bounded measurable function from $[0,+\infty)$ to $\mathbb{R}^{n}$ such that $T:=\inf \{t>0: \xi(t) \notin \Omega\}<+\infty$, and with cost functional

$$
J(x, q)=\int_{0}^{T} \delta(\xi(t), q(t)) d t+g(\xi(T)) .
$$

The dynamic programming equation associated to the control problem (2.10)-(2.11) is

$$
\sup _{|q| \leq 1}\{q D u(x)-\delta(x, q)\}=0 \quad x \in \Omega .
$$

This equation turns out to be equivalent to equation (1.2), in the sense that any viscosity sub or supersolution of equation (2.12) is also a viscosity sub- or supersolution of equation (1.2) and vice versa.

In the following we will assume that the boundary datum $g$ verifies the compatibility condition

$$
g(x)-g(y) \leq L(x, y) \quad \text { for any } x, y \in \partial \Omega .
$$

It is standard to show that, under hypothesis (2.13), $U$ is a viscosity solution of (1.2) and satisfies the boundary condition (1.3). Furthermore we have

Proposition 2.5 The value function $U$ is a singular supersolution of equation (1.2) in $\Omega$.

Theorem 2.4 and Proposition 2.5 now allow us to characterize the maximal solution of (1.2)-(1.3): Let $\mathcal{S}$ denote the set of functions $v \in U S C(\bar{\Omega})$ which are viscosity subsolutions of (1.2) and which satisfy $v \leq g$ on $\partial \Omega$. From Theorem 2.4 and Proposition 2.5 it follows that the value function $U$ of the control problem $(2.10)-(2.11)$ is the maximal element of $\mathcal{S}$, i.e. the maximal subsolution of problem (1.2)-(1.3). Moreover $U$ is a singular supersolution of (1.2) satisfying $U=g$ on $\partial \Omega$, hence it is the maximal solution. 
Remark 2.6 If $H$ is convex in $p$, then $U$ coincides with the value function of control problem with dynamics (2.10) and cost functional

$$
J(x, q)=\int_{0}^{T} f(\xi(t))+H^{*}(\xi(t), q(t)) d t+g(\xi(T)) .
$$

where $H^{*}(x, \cdot)$ denotes the Legendre transform of $H(x, \cdot)$, cp. [15]. Note, however, that $\delta(x, q)$ and $f(x)+H^{*}(x, q)$ in general do not coincide pointwise.

We conclude this section stating a particular case of a general stability theorem proved in [6] needed for the construction of the approximation scheme.

Proposition 2.7 Set $f_{\epsilon}(x)=\max \{f(x), \epsilon\}$ and let $u_{\epsilon}$ be the sequence of viscosity solutions of

$$
\begin{array}{ll}
H(x, D u)=f_{\epsilon}(x) & x \in \Omega, \\
u(x)=g(x) & x \in \partial \Omega .
\end{array}
$$

Then

$$
\lim _{\epsilon \rightarrow 0} u_{\epsilon}(x)=U(x)
$$

uniformly in $\bar{\Omega}$, where $U$ is the maximal solutions of (1.2)-(1.3).

Note that for any $\epsilon>0$ fixed, since $f_{\epsilon}>0$ in $\Omega$, problem (2.14) admits a unique viscosity solution. Moreover this solution is given by the value function of the control problem with dynamics (2.10) and cost functional

$$
J_{\epsilon}(x, q)=\int_{0}^{T} \delta_{\epsilon}(\xi(t), q(t)) d t+g(\xi(T))
$$

where $\xi(T) \in \partial \Omega$ and $\delta_{\epsilon}(x, q)$ is defined as $\delta(x, q)$ with $f_{\epsilon}$ instead of $f$.

We introduce some notations we will use in the following. We define

$$
M:=\sup \left\{\delta_{\epsilon}(x, q): x \in \Omega,|q|=1, \epsilon>0\right\} .
$$

Moreover, for $\epsilon>0$, we set

$$
\begin{aligned}
\omega_{\delta_{\epsilon}}(r) & :=\sup \left\{\left|\delta_{\epsilon}(x, p)-\delta_{\epsilon}(y, p)\right|:|p|=1,|x-y|<r\right\}, \\
\alpha(\epsilon) & :=\inf _{x \in \Omega,|q|=1} \delta_{\epsilon}(x, q) .
\end{aligned}
$$

Note that, for any $\epsilon>0, \alpha(\epsilon)>0$ and $\omega_{\delta_{\epsilon}}(r)$ is bounded by $\omega_{\delta}(r)=\sup \{|\delta(x, p)-\delta(y, p)|$ : $|p|=1,|x-y|<r\}$.

\section{The semidiscrete scheme}

Let us introduce the semidiscrete approximation scheme, obtained by discretizing in time the exit time control problem (2.10)-(2.15). For a fixed $\epsilon>0$, we choose a step in time $h \in(0,1)$ and define discrete dynamics by the recursive sequence

$$
\left\{\begin{array}{l}
x_{n+1}=x_{n}+h q_{n} \quad n \in \mathbb{N} \\
x_{0}=x
\end{array}\right.
$$


for $x \in \Omega,\left\{q_{n}\right\} \subset \mathbb{R}^{n}$ such that $\left|q_{n}\right|=1$.

The cost is given by

$$
J_{h \epsilon}\left(x, q_{n}\right)=\sum_{n=0}^{N-1} h \delta_{\epsilon}\left(x_{n}, q_{n}\right)+g\left(x_{N}\right)
$$

where

$$
N=\inf \left\{n \in \mathbb{N}: x_{n} \notin \Omega\right\}
$$

(we assume the convention that $\sum_{n=0}^{-1}=0$ ). The value function for this control problem is

$$
u_{h \epsilon}(x)=\inf \left\{J_{h \epsilon}\left(x, q_{n}\right):\left\{q_{n}\right\} \text { such that } N<+\infty\right\} .
$$

By a standard application of the discrete dynamic programming principle, the function $u_{h \epsilon}$ is a solution of the problem

$$
\left\{\begin{array}{lr}
u_{h \epsilon}(x)=\inf _{|q|=1}\left\{h \delta_{\epsilon}(x, q)+u_{h \epsilon}(x+h q)\right\} & x \in \Omega, \\
u(x)=g(x) & x \in \mathbb{R}^{N} \backslash \Omega .
\end{array}\right.
$$

The following result holds true

Proposition 3.1 There is a constant $C$ (independent of $h$ and $\epsilon$ ) such that

$$
\left|u_{h \epsilon}(x)\right| \leq C \quad \text { for any } x \in \bar{\Omega} .
$$

Moreover $u_{h \epsilon}$ is the unique bounded solution of (3.1).

Proof: We first observe that it is always possible to assume, by adding a constant, that $g \geq 0$. It follows that $u_{h \epsilon} \geq 0$. Moreover

$$
u_{h \epsilon}(x) \leq M \sup _{x \in \Omega} d(x, \partial \Omega)+\sup _{x \in \mathbb{R}^{n}} g(x)
$$

where $M$ is as in (2.16).

Let $v_{1}, v_{2}$ be two bounded solution of (3.1) and set $w_{i}(x)=1-e^{-v_{i}(x)}$, for $i=1,2$. Then $w_{i}$ satisfies

$$
\begin{cases}w_{i}(x)=\left[S w_{i}\right](x) & x \in \Omega \\ w_{i}=1-e^{-g(x)} & x \in \mathbb{R}^{N} \backslash \Omega,\end{cases}
$$

where

$$
[S \phi](x)=\inf _{|q|=1}\left\{1-h \delta_{\epsilon}(x, q)+e^{-h \delta_{\epsilon}(x, q)} \phi(x+h q)\right\} .
$$

It follows that

$$
\sup _{\Omega}\left|S w_{1}(x)-S w_{2}(x)\right| \leq \beta \sup _{\Omega}\left|w_{1}(x)-w_{2}(x)\right|
$$

with $\beta=e^{-h \alpha(\epsilon)}<1$, and $w_{1}=w_{2}=g$ in $\mathbb{R}^{N} \backslash \Omega$.

We conclude that for any $\epsilon>0$ and $h>0$ there exists at most one bounded solution of (3.3) and therefore of problem (3.1). This solution is given by $u_{h \epsilon}$.

Remark 3.2 If we discretized the control problem (2.10)-(2.11) directly (which corresponds to setting $\epsilon=0$ in the previous approximation scheme), the resulting approximating equation does not have a unique bounded solution, similarly to what happens in problem (1.2)-(1.3). This causes the drawback that any algorithm designed to solve that approximating equation could not converge to the maximal viscosity solution and, in any case, displays high numerical instability (see [12]). 


\section{Convergence of the semidiscrete scheme}

In this section, we prove the convergence of the approximation scheme introduced in the previous section to the maximal solution of (1.2)-(1.3).

Given a locally uniformly bounded sequence of functions $v_{\epsilon}: \bar{\Omega} \rightarrow \mathbb{R}, \epsilon>0$, we set

$$
\begin{aligned}
\liminf _{\epsilon \rightarrow 0} v_{\epsilon}(x) & =\inf \left\{\liminf _{\epsilon \rightarrow 0} v_{\epsilon}\left(x_{\epsilon}\right): x_{\epsilon} \rightarrow x, x_{\epsilon} \in \bar{\Omega}\right\} \\
\limsup _{\epsilon \rightarrow 0} v_{\epsilon}(x) & =\sup \left\{\limsup _{\epsilon \rightarrow 0} v_{\epsilon}\left(x_{\epsilon}\right): x_{\epsilon} \rightarrow x, x_{\epsilon} \in \bar{\Omega}\right\}
\end{aligned}
$$

for any $x \in \bar{\Omega}$. The functions $\liminf _{\epsilon \rightarrow 0} v_{\epsilon}(x)$ and $\limsup _{\epsilon \rightarrow 0} v_{\epsilon}(x)$ are, respectively, l.s.c. and u.s.c. in $\bar{\Omega}$.

Lemma 4.1 Let $u_{h \epsilon}$ be a sequence of solutions of (3.1) and assume that $h=h(\epsilon)$ is such that

$$
\frac{\omega_{\delta_{\epsilon}}(h)}{\alpha(\epsilon)} \rightarrow 0 \quad \text { as } \epsilon \rightarrow 0^{+}
$$

Then

$$
\underline{u}(x)=\liminf _{\epsilon \rightarrow 0} u_{h \epsilon}(x) \quad x \in \Omega
$$

is a singular supersolution of (1.2).

Proof: Because of (3.2), the function $\underline{u}$ is well defined in $\bar{\Omega}$. Let $\phi: \Omega \rightarrow \mathbb{R}$ be $L$-subtangent to $\underline{u}$ at $x_{0} \in \Omega$. It is possible to assume without loss of generality (see [6], Proposition 5.1) that $\phi$ is a strict $L$-subtangent to $\underline{u}$ at $x_{0}$.

Employing a standard argument in viscosity solution theory, we find a sequence $x_{\epsilon}$ of minimum points for $u_{h \epsilon}-\phi$ such that $L\left(x_{0}, x_{\epsilon}\right) \rightarrow 0$ as $\epsilon$ tends to $0^{+}$. Then

$$
\begin{aligned}
0 & =\sup _{|q|=1}\left\{-\frac{u_{h \epsilon}\left(x_{\epsilon}+h q\right)-u_{h \epsilon}\left(x_{\epsilon}\right)}{h}-\delta_{\epsilon}\left(x_{\epsilon}, q\right)\right\} \leq \\
& \leq \max _{|q|=1}\left\{-\frac{\phi\left(x_{\epsilon}+h q\right)-\phi\left(x_{\epsilon}\right)}{h}-\delta_{\epsilon}\left(x_{\epsilon}, q\right)\right\}= \\
& =-\frac{\phi\left(x_{\epsilon}+h q_{\epsilon}\right)-\phi\left(x_{\epsilon}\right)}{h}-\delta_{\epsilon}\left(x_{\epsilon}, q_{\epsilon}\right)
\end{aligned}
$$

for some $q_{\epsilon}$ with $\left|q_{\epsilon}\right|=1$.

From the Mean Value Theorem for Lipschitz continuous functions (see Clarke [7]), there exist $\theta_{\epsilon} \in(0,1)$ and $p_{\epsilon} \in \partial \phi\left(x_{\epsilon}+h \theta_{\epsilon} q_{\epsilon}\right)$ such that

$$
\phi\left(x_{\epsilon}+h q_{\epsilon}\right)-\phi\left(x_{\epsilon}\right)=h p_{\epsilon} q_{\epsilon} .
$$

Set $\bar{x}_{\epsilon}=x_{\epsilon}+h q_{\epsilon}$. Substituting (4.3) into (4.2), we get

$$
0 \leq p_{\epsilon} q_{\epsilon}-\delta_{\epsilon}\left(x_{\epsilon}, q_{\epsilon}\right) \leq p_{\epsilon} q_{\epsilon}-\delta_{\epsilon}\left(\bar{x}_{\epsilon}, q_{\epsilon}\right)+\omega_{\delta_{\epsilon}}(h) .
$$

Observe that $\bar{x}_{\epsilon} \notin K$, otherwise, since $\partial \phi(x)=\{0\}$ on $K$, we should have $p_{\epsilon}=0$ and from $(4.4)$

$$
0 \leq-\delta_{\epsilon}\left(x_{\epsilon}, q_{\epsilon}\right)
$$


which is impossible since $\delta_{\epsilon}$ is strictly positive in $\Omega$.

Let $\bar{q}_{\epsilon}=q_{\epsilon} / \delta_{\epsilon}\left(\bar{x}_{\epsilon}, q_{\epsilon}\right)$. By the homogeneity of $\delta_{\epsilon}(x, q)$ with respect to $q$, we have $\bar{q}_{\epsilon} \in\{q \in$ $\left.\mathbb{R}^{N}: \delta_{\epsilon}\left(\bar{x}_{\epsilon}, q\right) \leq 1\right\}$. Dividing (4.4) by $\delta_{\epsilon}\left(\bar{x}_{\epsilon}, q_{\epsilon}\right)$ and recalling (2.6), we get

$$
\begin{aligned}
0 & \leq p_{\epsilon} \bar{q}_{\epsilon}-1+\frac{\omega_{\delta_{\epsilon}}(h)}{\alpha(\epsilon)} \leq \sup _{\delta_{\epsilon}\left(\bar{x}_{\epsilon}, q\right) \leq 1}\left\{p_{\epsilon} q\right\}-1+\frac{\omega_{\delta_{\epsilon}}(h)}{\alpha(\epsilon)} \leq \\
& \leq \sup _{\delta\left(\bar{x}_{\epsilon}, q\right) \leq 1}\left\{p_{\epsilon} q\right\}-1+\frac{\omega_{\delta_{\epsilon}}(h)}{\alpha(\epsilon)} \leq \rho\left(\bar{x}_{\epsilon}, p_{\epsilon}\right)-1+\frac{\omega_{\delta_{\epsilon}}(h)}{\alpha(\epsilon)} .
\end{aligned}
$$

Since the sequence $\bar{x}_{\epsilon}$ belongs to $\Omega \backslash K$ and $L\left(x_{0}, \bar{x}_{\epsilon}\right) \rightarrow 0$, as $\epsilon \rightarrow 0^{+}$, we conclude, thanks to hypothesis (4.1), that $\underline{u}$ is a singular supersolution of (1.2).

Theorem 4.2 Assume that either

$$
g \equiv 0
$$

or

$$
\Omega \text { is convex. }
$$

If $u_{h \epsilon}$ is a sequence of solutions of problem (3.1) and $h=h(\epsilon)$ satisfies the assumption (4.1), then

$$
\lim _{\epsilon \rightarrow 0} u_{h \epsilon}(x)=U(x) \quad \text { uniformly in } \bar{\Omega},
$$

where $U$ is the maximal solution of problem (1.2)-(1.3).

Proof: We set

$$
\begin{aligned}
& \underline{u}(x)=\liminf _{\epsilon \rightarrow 0} u_{h \epsilon}(x), \\
& \bar{u}(x)=\limsup _{\epsilon \rightarrow 0} u_{h \epsilon}(x)
\end{aligned}
$$

for $x \in \bar{\Omega}$. These function are well defined because of (3.2).

From Proposition 4.1, it follows that $\underline{u}$ is a singular supersolution of equation (1.2). Moreover it is standard to show that $\bar{u}$ is a subsolution of (2.12) and therefore of (1.2) in $\Omega$ (see, f.e., [1] or [2]). If we show that $\underline{u} \geq \bar{u}$ on $\partial \Omega$, then Theorem 2.4 and Proposition 2.5 imply that $\underline{u}=\bar{u}=U$ in $\bar{\Omega}$ and therefore (4.7).

We will show that

$$
\bar{u}(x) \leq g(x) \leq \underline{u}(x) \quad \text { for any } x \in \partial \Omega .
$$

To show that $\bar{u}(x) \leq g(x)$ on $\partial \Omega$, we need an estimate on the behavior of $u_{h \epsilon}$ in a neighborhood of $\partial \Omega$. Let $\eta>0$ be sufficiently small and set $\Omega_{\eta}=\{x \in \Omega: d(x, \partial \Omega)<\eta\}$. For $x \in \Omega_{\eta}$, let $y \in \partial \Omega$ be such that $d(x, \partial \Omega)=|y-x|$. Define a control law $\left\{q_{n}\right\}$ for the discrete control problem by

$$
q_{n}=\frac{x-y}{|x-y|} \quad n \in \mathbb{N}
$$

and, denoted by $x_{n}$ the corresponding discrete trajectory, let $N=\inf \left\{n>0: x_{n} \notin \Omega\right\}$. Observing that $N h \leq|y-x|$, we get

$$
\begin{gathered}
u_{h \epsilon}(x) \leq \sum_{n=0}^{N-1} h \delta_{\epsilon}\left(x_{n}, q_{n}\right)+g\left(x_{N}\right) \leq \\
\leq M|y-x|+g(y)+\omega_{g}(h) .
\end{gathered}
$$


where $M$ is as in (2.16) and $\omega_{g}$ is a modulus of continuity of $g$. If $x_{0} \in \partial \Omega$ and $x_{\epsilon} \in \bar{\Omega}$ is a sequence converging to $x_{0}$, we have either $u_{h \epsilon}\left(x_{\epsilon}\right)=g\left(x_{\epsilon}\right)$, if $x_{\epsilon} \in \partial \Omega$, or

$$
u_{h \epsilon}\left(x_{\epsilon}\right) \leq M\left|y_{\epsilon}-x_{\epsilon}\right|+g\left(y_{\epsilon}\right)+\omega_{g}(h)
$$

if $x_{\epsilon} \in \Omega$, where $y_{\epsilon} \in \partial \Omega$ is such that $d\left(x_{\epsilon}, \partial \Omega\right)=\left|x_{\epsilon}-y_{\epsilon}\right|$. Since also $y_{\epsilon}$ converges to $x_{0}$, we get $\bar{u}\left(x_{0}\right) \leq g\left(x_{0}\right)$ on $\partial \Omega$.

To get the other inequality in (4.8), if $g \equiv 0$, then $u_{h \epsilon}(x) \geq 0$ in $\bar{\Omega}$ and therefore $\underline{u} \geq 0$ on $\partial \Omega$.

If (4.6) holds, by adding a constant, we can always assume that $g \geq 0$.

For $x \in \bar{\Omega}$, let $q_{n}$ be an $\eta$-optimal control for $u_{h \epsilon}(x), x_{n}$ the corresponding discrete trajectory and $N$ the exit time from $\Omega$. Since

$$
\alpha(\epsilon) N h \leq \sum_{n=0}^{N-1} h \delta_{\epsilon}\left(x_{n}, q_{n}\right)+g\left(x_{N}\right) \leq u_{h \epsilon}(x)+\eta
$$

we have

$$
N h \leq \frac{C+1}{\alpha(\epsilon)}
$$

with $C$ as in (3.2).

Let $q(t)$ be a control law for the continuous problem obtained by setting $q(t)=q_{i}$ for $t \in[i h,(i+1) h), i=0,1, \ldots, N-1$. If $\xi(t)$ and $T$ are respectively the trajectory and the exit time corresponding to $q(t)$, we have

$$
\begin{aligned}
u_{\epsilon}(x) & \leq \int_{0}^{N h} \delta_{\epsilon}(\xi(t), q(t)) d t+g(\xi(T)) \leq \\
& \leq \sum_{n=0}^{N-1} h\left(\delta_{\epsilon}\left(x_{n}, q_{n}\right)+\omega_{\delta_{\epsilon}}(h)\right)+g\left(x_{N}\right)+\omega_{g}\left(\left|\xi(T)-x_{N}\right|\right) \leq \\
& \leq u_{h \epsilon}(x)+\eta+C \frac{\omega_{\delta_{\epsilon}}(h)}{\alpha(\epsilon)}+\omega_{g}(h),
\end{aligned}
$$

where the estimate $\left|\xi(T)-x_{N}\right| \leq h$ holds because of the convexity of $\Omega$. Since $u_{\epsilon}(x)=g(x)$ for any $x \in \partial \Omega$ and the assumption (4.1) is satisfied, from (4.9) we easily get other inequality in (4.8).

Remark 4.3 For the Eikonal equation (1.1) we have $\delta_{\epsilon}(x, q)=f_{\epsilon}(x)|q|$ and therefore condition (4.1) reduces to

$$
\frac{\omega_{f}(h)}{\epsilon} \rightarrow 0 \quad \text { as } \epsilon \rightarrow 0^{+}
$$

where $\omega_{f}$ is the modulus of continuity of the function $f$ on $\bar{\Omega}$.

\section{Discretization error for the fully discrete scheme}

In this section we will discuss a fully discrete scheme derived from the semidiscrete one as developed in the previous sections. In order to simplify the calculations we assume that 
the function $g$ defining the boundary condition is uniformly Lipschitz with constant $L_{g}$, and that the domain $\Omega$ is convex.

We will introduce a space discretization which transforms (3.1) into a finite dimensional problem. For this purpose we choose a grid $\Gamma$ covering $\bar{\Omega}$ consisting of simplices $S_{j}$ with nodes $x_{i}$ and look for the solution of (3.1) in the space

$$
W:=\left\{w \in C(\bar{\Omega}) \mid \nabla w \equiv \text { const on } S_{j}\right\}
$$

of piecewise linear functions on $\Gamma$. By the parameter $k$ we denote the maximal diameter of the simplices $S_{j}$. For simplicity we assume that the boundary of the gridded domain coincides with the boundary of $\Omega$. (In the general case we can always achieve an error scaling linearly with the distance between these two boundaries due to the fact that $g$ is Lipschitz).

Thus we end up with the fully discrete scheme

$$
u_{\epsilon, h}^{k}\left(x_{i}\right)=\inf _{|q|=1}\left\{h \delta_{\epsilon}\left(x_{i}, q\right)+u_{\epsilon, h}^{k}\left(x_{i}+h q\right)\right\}
$$

for all nodes $x_{i} \in \Omega$ with the boundary condition $u_{\epsilon, h}^{k}\left(x_{i}\right)=g\left(x_{i}\right)$ for the nodes $x_{i} \notin \Omega$ and linear interpolation between the nodes.

Note that there exists a unique bounded solution of (5.1). The boundedness of any solution of (5.1) follows from the fact that

$$
u_{\epsilon, h}^{k}\left(x_{i}\right) \leq h \delta_{\epsilon}\left(x_{i}, q\right)+u_{\epsilon, h}^{k}\left(x_{i}+h q\right)
$$

holds for any $q \in \mathbb{R}^{n}$ with $|q|=1$. Thus we can always choose $q$ such that $u_{\epsilon, h}^{k}\left(x_{i}+h q\right)$ depends on nodes which are closer to the boundary $\partial \Omega$ than $x_{i}$ and (if $h<k$ ) on $x_{i}$ itself, but with a weight strictly less than one. Since the value in the boundary nodes is bounded we obtain boundedness for each node by induction.

Due to the boundedness the existence of a unique solution $u_{\epsilon, h}^{k}$ is now easily proved by applying the Kruzkov transformation

$$
v(x)=1-e^{-u_{\epsilon, h}^{k}(x)}
$$

as in the proof of Proposition 3.1.

Note that the function $\delta_{\epsilon}$ appearing in the scheme is defined implicitely via $H$ and $f_{\epsilon}$. In order to solve the scheme we assume that we can compute this function analytically as e.g. in Example 2.1. (In the case of a convex Hamiltonian one may alternatively use a numerical approximation of the integrand from Remark 2.6 via the Legendre transform as given e.g. in [10]. Note, however, that this procedure yields a different cost function than in the following analysis.)

We will now start by estimating the discretization error $\left|u_{\epsilon}(x)-u_{\epsilon, h}^{k}(x)\right|, x \in \Omega$. Since we allow nonconstant boundary conditions we introduce the following auxiliary functions which will be useful for the estimation of the error. 
Definition 5.1 For each point $x \in \Omega$ we define

$$
w_{1}(x)=u_{\epsilon}(x)-u_{\epsilon}(\xi(T))
$$

where $\xi(\cdot)$ is an optimal path for the initial value $x$ and $\xi(T) \in \partial \Omega$.

For each node $x_{i}$ of the grid pick a control $q_{i}$ minimizing (5.1) and let $w_{2} \in W$ be the unique solution of

$$
w_{2}\left(x_{i}\right)=h \delta_{\epsilon}\left(x_{i}, q_{i}\right)+w_{2}\left(x_{i}+h q_{i}\right)
$$

with the boundary condition $w_{2}(x)=0$ and interpolation between the nodes.

Finally we define $w(x)=\max \left\{w_{1}(x), w_{2}(x)\right\}$.

Remark 5.2 The existence of optimal paths follows from the continuous dependence of the functional $J(x, q)$ from the control function $q$ using the weak ${ }^{*}$-metric (as defined for control functions e.g. in [9]), using the Gronwall Lemma as in [8, Proof of Lemma 3.4(ii)] and the structure of $\delta_{\epsilon}$. Note that the a-priori boundedness of the length of approximately optimal trajectories - following from the positivity of $\delta_{\epsilon}$ - is crucial for this continuous dependence. Thus in general the existence of optimal trajectories does not hold for the non-regularized problem since there for any sequence of approximately optimal trajectories the length of these trajectories may grow unbounded when we restrict $|q(t)|=1$ for all $t \geq 0$.

Note that we do not require uniqueness of the optimal paths in Definition 5.1. In the case that there is no unique optimal path we may use one that minimizes $w_{1}$.

Definition 5.1 defines functions which are 0 at $\partial \Omega$ and away from $\partial \Omega$ essentially grow like $u_{\epsilon}$ and $u_{\epsilon, h}^{k}$, respectively. More precisely we have that

$$
w_{1}(\xi(t))-w_{1}(x)=u_{\epsilon}(\xi(t))-u_{\epsilon}(x)
$$

and

$$
w_{2}\left(x_{i}+h q_{i}\right)-w_{2}\left(x_{i}\right)=u_{\epsilon, h}^{k}\left(x_{i}+h q_{i}\right)-u_{\epsilon, h}^{k}\left(x_{i}\right)
$$

for $\xi(\cdot)$ and $q_{i}$ as used in the definition.

Note that in particular if $g(x) \equiv c$ is constant we obtain

$$
w(x)=\max \left\{u_{\epsilon}(x), u_{\epsilon, h}^{k}(x)\right\}-c .
$$

Using this $w$ we can give the following estimate for the discretization error.

Proposition 5.3 Let $u_{\epsilon, h}^{k} \in W$ be the unique solution of (5.1). Then the estimate

$$
\left|u_{\epsilon}(x)-u_{\epsilon, h}^{k}(x)\right| \leq C\left[\frac{k \omega_{\delta_{\epsilon}}(k)}{h \alpha(\epsilon)}+\frac{k}{\beta(\epsilon) h}+\frac{\omega_{\delta_{\epsilon}}(h)}{\alpha(\epsilon)}\right] w(x)+\left(M+L_{g}\right) h+L_{g} k
$$

holds for each $x \in \bar{\Omega}$ and for all sufficiently small $k>0$ and $h>0$ with $M, \omega_{\delta_{\epsilon}}$ and $\alpha$ as defined in (2.16)-(2.18),

$$
\beta(\epsilon)=\inf _{x \in \Omega,|q|=|p|=1} \frac{\delta_{\epsilon}(x, p)}{\delta_{\epsilon}(x, q)},
$$

and some constant $C$ independent from $\epsilon, h$ and $k$. 
The proof can be found in the appendix.

Remark 5.4 (i) Note that estimate (5.3) is stronger than the usual $L_{\infty}$ estimate since essentially the error scales with the function $w(x)$ being 0 at $\partial \Omega$. The reason for this behaviour origins in the fact that the error is estimated along the optimal trajectories whose length depends on the optimal value.

(ii) The constant $\alpha(\epsilon)$ essentially depends on the growth of $H$ in $|p|$, e.g. in Example 2.1 we have $\alpha(\epsilon)=\psi^{-1}(\epsilon)$. The constant $\beta(\epsilon)$ is determined by the difference between $H(x, p)$ and $H(x, q)$ for $|p|=|q|$. In particular if $H(x, p) \in\left[C_{1}|p|^{\gamma}, C_{2}|p|^{\gamma}\right]$ we have that $\beta(\epsilon) \geq C_{1} / C_{2}$ independently from $\epsilon$. Finally, $\omega_{\delta}$ (which gives a bound for $\omega_{\delta_{\epsilon}}$ for all $\epsilon>0$ ) combines the continuity properties of $H$ and $f$, i.e. in Example 2.1 we have that $\omega_{\delta}(r)=\omega_{\psi^{-1} \circ f}(r)$.

(iii) Note that the requirement on $h$ ensuring the convergence of the fully discrete scheme is that $\omega_{\delta_{\epsilon}}(h) / \alpha(\epsilon) \rightarrow 0$ as $\epsilon \rightarrow 0$, thus it is consistent with condition (4.1) for the convergence of the semidiscrete scheme.

(iv) The appearance of the value $\alpha(\epsilon)$ in the denominator in (5.3) is due to the fact that here we implicitely included the worst case, i.e. that the length of the optimal trajectories may grow like $1 / \alpha(\epsilon)$ for $\alpha(\epsilon) \rightarrow 0$. Since this is not necessarily the case in many practical examples one can expect better convergence behaviour for $\alpha(\epsilon) \rightarrow 0$.

(v) A particular nice formulation of estimate (5.3) can be obtained if we consider the Eikonal equation (1.1) (implying $\beta(\epsilon)=1$ and $\alpha(\epsilon)=\epsilon$ ), assume that $f$ is uniformly Lipschitz (implying $\omega_{\delta_{\epsilon}}(h) \leq L_{f} h$ ) and impose a homogeneous boundary condition, i.e. $g \equiv c$ (implying $L_{g}=0$ ). In this case the estimate becomes

$$
\left|u_{\epsilon}(x)-u_{\epsilon, h}^{k}(x)\right| \leq C\left[\frac{k^{2}}{h \epsilon}+\frac{k}{h}+\frac{h}{\epsilon}\right]\left(\max \left\{u_{\epsilon}(x), u_{\epsilon, h}^{k}(x)\right\}-c\right)+C h
$$

for some constant $C>0$ independent from $\epsilon, h$ and $k$. In particular this implies convergence of the scheme if $\varepsilon \rightarrow 0, h / \varepsilon \rightarrow 0$ and $k / h \rightarrow 0$.

We will now turn to the discussion of the error obtained when equation (1.2) is replaced by equation (2.14), i.e. the error introduced by the regularization of the problem.

Proposition 2.7 already implies that $u_{\varepsilon}$ converges to $U$, where $U$ is the maximal subsolution of (1.2). Unfortunately, in general this convergence can be arbitrary slow. In the optimal control interpretation this is due to the fact that the length of approximately optimal trajectories may grow unbounded as the approximation gets better and better. Since these long pieces of the trajectories can only appear in regions where $f$ is sufficiently small (otherwise the cost would be large contradicting the approximate optimality), we can derive an estimate for the regularization error by defining a criterion for the sets where $f$ is small which in turn gives a bound on the length of approximately optimal trajectories.

The following definition is our main tool for this purpose.

Definition 5.5 Let $B \subset \mathbb{R}^{d}$ be a compact set. For each connected component $B_{i}$ of $B$ we define the inner diameter $d\left(B_{i}\right)$ by

$$
d\left(B_{i}\right):=\sup _{x, y \in B_{i}} d_{B_{i}}(x, y)
$$


where

$$
d_{B_{i}}(x, y):=\inf \left\{\begin{array}{l|l}
T \geq 0 & \begin{array}{l}
\exists \xi(t) \in W^{1, \infty}\left([0, T], B_{i}\right) \\
\text { s.t. } \xi(0)=x, \xi(T)=y, \\
|\dot{\xi}(t)|=1 \text { a.e. }
\end{array}
\end{array}\right\}
$$

and for $B$ we define the inner diameter by

$$
d(B):=\sum_{B_{i}} d\left(B_{i}\right)
$$

where the sum is taken over all connected components of $B$.

Using this definition we can state the following estimate for the regularization error.

Proposition 5.6 Let $U$ be the maximal subsolution of (1.2) and let $u_{\epsilon}$ be the unique viscosity solution of (2.14).

Then the estimate

$$
\left\|U-u_{\epsilon}\right\|_{\infty} \leq c(\epsilon) d\left(K_{\epsilon}\right)
$$

holds where $K_{\epsilon}:=\{x \in \Omega \mid f(x) \leq \epsilon\}$ and $c(\epsilon)=\sup _{x \in K_{\epsilon},|q|=1} \delta_{\epsilon}(x, q)$.

The proof can be found in the appendix.

Here the constant $c(\epsilon)$ depends only on the sets $\mathcal{Z}_{\epsilon}(x)=\left\{p: H(x, p) \leq f_{\epsilon}(x)\right\}$, i.e. on $\epsilon$ and on the Hamiltonian $H$. In fact an easy calculation shows that

$$
c(\epsilon)=\sup _{x \in K_{\epsilon}, p \in \mathcal{Z}_{\epsilon}(x)}|p|=\sup _{x \in K_{\epsilon}, p \in \mathbb{R}^{n}, H(x, p) \leq \epsilon}|p| .
$$

Thus e.g. the estimate $c(\epsilon) \leq C \epsilon^{\gamma}$ for some constants $C, \gamma>0$ and all $\epsilon>0$ sufficiently small holds if $H(x, p) \geq(|p| / C)^{1 / \gamma}$ for all $x \in K_{\epsilon}$, all $\epsilon>0$ sufficently small and all $p \in \mathbb{R}^{n}$ with $|p|$ sufficiently small. In particular for the Eikonal equation (1.1) this implies $c(\epsilon)=\epsilon$.

Observe that if $f$ is piecewise polynomial then $d\left(K_{\varepsilon}\right)$ is bounded for all $\epsilon>0$ and hence convergence with order $c(\epsilon)$ follows for $\epsilon \rightarrow 0$. Piecewise polynomial maps are in particular interesting since they include the case where $f$ is obtained from experimental data by some polynomial interpolation (e.g. using piecewise linear interpolations, multidimensional splines...).

The following theorem now gives the full a-priori estimates for the approximation error of the whole numerical approximation.

Theorem 5.7 Let $U$ be the maximal subsolution of (1.2) and let $u_{\epsilon, h}^{k}$ be the unique solution of the numerical scheme (5.1).

Then the estimate

$$
\left|U(x)-u_{\epsilon, h}^{k}(x)\right| \leq c(\epsilon) d\left(K_{\epsilon}\right)+C\left[\frac{k \omega_{\delta_{\epsilon}}(k)}{h \alpha(\epsilon)}+\frac{k}{\beta(\epsilon) h}+\frac{\omega_{\delta_{\epsilon}}(h)}{\alpha(\epsilon)}\right] w(x)+\left(M_{\delta_{\epsilon}}+2 L_{g}\right) h+L_{g} k
$$

holds for each $x \in \bar{\Omega}$ and the constants from the Propositions 5.3 and 5.6. 
Proof: Follows immediately from the Propositions 5.3 and 5.6.

Remark 5.8 (i) A possible modification of the scheme can be made if we allow smaller time steps at the boundary $\partial \Omega$, i.e. for $x_{i} \in \Omega$ and $x_{i}+h q \notin \bar{\Omega}$ we use the restricted time step

$$
\bar{h}=\sup \left\{h \in[0, h] \mid x_{i}+h q \in \Omega\right\} .
$$

Although slightly more difficult to implement this modification usually gives better numerical results. The proof of Proposition 5.3 also applies to this modified scheme.

(ii) Due to the structural similarity of the scheme described in this section with the scheme considered in [13], the adaptive grid scheme developed there can also be applied here. Similar convergence results as in [13] can be obtained for our scheme using the technique from the proof of Proposition 5.3.

\section{Appendix: Proof of the Propositions 5.3 and 5.6}

In order to prove Proposition 5.3 we will first state a useful estimate for the local error along the functional.

Lemma 6.1 For each measurable $q(\cdot)$ with $|q(t)|=1$ for almost all $t \in[0, h]$ and the path $\xi(\cdot)$ with $\dot{\xi}(t)=q(t)$ and $\xi(t) \in \bar{\Omega}$ for all $t \in[0, h]$ there exists $p \in \mathbb{R}^{n}$ with $|p|=1$ such that

$$
h \delta(\xi(0), p) \leq \int_{0}^{h} \delta_{\epsilon}(\xi(t), q(t)) d t+h \omega_{\delta_{\epsilon}}(h)
$$

and

$$
\xi(0)+h p=\xi(h) .
$$

Conversely, for each $p \in \mathbb{R}^{n}$ with $|p|=1$ and each $x \in \Omega$ with $x+h p \in \bar{\Omega}$ there exists a measurable function $q(\cdot)$ with $|q(t)|=1$ for all $t \geq 0$ such that

$$
\int_{0}^{h} \delta_{\epsilon}(\xi(t), q(t)) d t \leq h \delta(x, p)+h \omega_{\delta_{\epsilon}}(h)
$$

and

$$
\xi(0)=x, \quad \xi(h)=x+h p
$$

where $\dot{\xi}(t)=q(t)$ and $\xi(t) \in \bar{\Omega}$ for all $t \in[0, h]$.

Proof: The convexity of $\delta_{\epsilon}$ in the second argument implies

$$
\delta_{\epsilon}\left(x, \int_{0}^{h} q(t) d t\right) \leq \int_{0}^{h} \delta_{\epsilon}(x, q(t)) d t .
$$

Hence by defining

$$
p=\frac{1}{h} \int_{0}^{h} q(t) d t
$$

the first assertion immediately follows from the continuity of $\delta_{\epsilon}$ which is measured by $\omega_{\delta_{\epsilon}}$. 
The second assertion follows directly from the continuity of $\delta_{\epsilon}$ setting $q(t) \equiv p$ and using the convexity of $\bar{\Omega}$.

\section{Proof of Proposition 5.3}

We start giving some preliminary estimates.

First note that the error at the boundary can be estimated by

$$
\left|u_{\epsilon, h}^{k}(x)-u_{\epsilon}(x)\right| \leq L_{g} k
$$

which simply follows from the Lipschitz property of $g$.

Furthermore it is easy to see that on each element $S_{j}$ of the grid we can estimate

$$
\left|u_{\epsilon}(x)-u_{\epsilon}(y)\right| \leq \sup _{z \in S_{j},|q|=1} \delta_{\epsilon}(z, q) k
$$

for each two points $x, y \in S_{j}$. We abbreviate $\kappa_{j}=\sup _{z \in S_{j},|q|=1} \delta_{\epsilon}(z, q)$.

We show the estimate (5.3) by estimating seperately the quantities $u_{\epsilon, h}^{k}(x)-u_{\epsilon}(x)$ and $u_{\epsilon}(x)-u_{\epsilon, h}^{k}(x)$. First, we consider $u_{\epsilon, h}^{k}(x)-u_{\epsilon}(x)$.

Observe that for any $\gamma>0$ there exists an $\eta \geq 0$ such that

$$
u_{\epsilon, h}^{k}(x)-u_{\epsilon}(x) \leq \eta\left(w_{1}(x)+\gamma\right)+\left(M_{\delta_{\epsilon}}+L_{g}\right) h+L_{g} k \quad \text { for all } x \in \bar{\Omega}
$$

which easily follows from the fact that $w_{1} \geq 0$ and $u_{\epsilon, h}^{k}$ is bounded.

Now we fix some arbitrary $\gamma>0$ and choose $\eta \geq 0$ to be minimal with (6.3). If $\eta=0$ the assertion immediately follows. Otherwise if $\eta>0$ by the continuity of the functions and the compactness of $\bar{\Omega}$ we can conclude that there exists $x^{*} \in \bar{\Omega}$ such that

$$
u_{\epsilon, h}^{k}\left(x^{*}\right)-u_{\epsilon}\left(x^{*}\right)=\eta\left(w_{1}\left(x^{*}\right)+\gamma\right)+\left(M_{\delta_{\epsilon}}+L_{g}\right) h+L_{g} k .
$$

Now consider the element $S_{j}$ containing $x^{*}$. We can write $x^{*}=\sum_{i \in I} \lambda_{i} x_{i}$ where the $x_{i}$ are the nodes of $S_{j}$ and the $\lambda_{i}$ are nonnegative coefficients with $\sum_{i \in I} \lambda_{i}=1$.

Using estimate (6.2) we obtain

$$
u_{\epsilon, h}^{k}\left(x^{*}\right)-u_{\epsilon}\left(x^{*}\right) \leq \sum_{i \in I} \lambda_{i}\left(u_{\epsilon, h}^{k}\left(x_{i}\right)-u_{\epsilon}\left(x_{i}\right)\right)+\kappa_{j} k .
$$

Now for each of the nodes we distinguish three cases.

(i) $x_{i} \in \partial \Omega:$ By (6.1) this implies

$$
u_{\epsilon, h}^{k}\left(x_{i}\right)-u_{\epsilon}\left(x_{i}\right) \leq L_{g} k
$$

(ii) $x_{i} \in \Omega$ and for the optimal path $\xi_{i}(\cdot)$ with $\xi_{i}(0)=x_{i}$ from Definition 5.1 there exists a time $\tau_{i} \in(0, h]$ such that

$$
u_{\epsilon}\left(x_{i}\right)=\int_{0}^{\tau_{i}} \delta_{\epsilon}\left(\xi_{i}(t), q_{i}(t)\right) d t+g\left(\xi_{i}\left(\tau_{i}\right)\right)
$$


where $\xi_{i}\left(\tau_{i}\right) \in \partial \Omega$ and $\dot{\xi}_{i}(t)=q_{i}(t)$. In this case by the convexity of $\bar{\Omega}$ we can conclude that there exists $p \in \mathbb{R}^{n}$ with $|p|=1$ and $x_{i}+h p \notin \Omega$ such that $\left|x_{i}+h p-\xi_{i}\left(\tau_{i}\right)\right| \leq h$. Thus we obtain

$$
u_{\epsilon, h}^{k}\left(x_{i}\right)-u_{\epsilon}\left(x_{i}\right) \leq\left(M_{\delta_{\epsilon}}+L_{g}\right) h+L_{g} k
$$

(iii) $x_{i} \in \Omega$ and for the optimal path $\xi_{i}(\cdot)$ with $\xi_{i}(0)=x_{i}$ from Definition 5.1 the equality

$$
u_{\epsilon}\left(x_{i}\right)=\int_{0}^{h} \delta_{\epsilon}\left(\xi_{i}(t), q_{i}(t)\right) d t+u_{\epsilon}\left(\xi_{i}(h)\right)
$$

holds where $\xi_{i}(0)=x_{i}, \xi_{i}(h) \notin \partial \Omega$ and $\dot{\xi}_{i}(t)=q_{i}(t)$.

In this case Lemma 6.1 and the definition of $u_{\epsilon, h}^{k}$ imply

$$
u_{\epsilon, h}^{k}\left(x_{i}\right)-u_{\epsilon}\left(x_{i}\right) \leq h \omega_{\delta_{\epsilon}}(h)+u_{\epsilon, h}^{k}\left(\xi_{i}(h)\right)-u_{\epsilon}\left(\xi_{i}(h)\right)
$$

where by (6.8) we can estimate

$$
u_{\epsilon}\left(\xi_{i}(h)\right) \leq u_{\epsilon}\left(x_{i}\right)-h \inf _{|q|=1} \delta_{\epsilon}\left(x_{i}, q\right)+h \omega_{\delta_{\epsilon}}(h)
$$

and by Definition 5.1 thus also

$$
w_{1}\left(\xi_{i}(h)\right) \leq w_{1}\left(x_{i}\right)-h \inf _{|q|=1} \delta_{\epsilon}\left(x_{i}, q\right)+h \omega_{\delta_{\epsilon}}(h) .
$$

Taking into account that the coefficients in (6.5) sum up to 1 we derive

$$
\sum_{i \in I} \lambda_{i}\left(u_{\epsilon, h}^{k}\left(x_{i}\right)-u_{\epsilon}\left(x_{i}\right)\right) \leq\left(M_{\delta_{\epsilon}}+L_{g}\right) h+L_{g} k+h \omega_{\delta_{\epsilon}}(h)+u_{\epsilon, h}^{k}\left(\xi_{i}(h)\right)-u_{\epsilon}\left(\xi_{i}(h)\right)
$$

and combining $(6.3),(6.4),(6.5),(6.10)$ and $(6.11)$ we obtain

$$
\eta\left(w_{1}\left(x^{*}\right)+\gamma\right) \leq h \omega_{\delta_{\epsilon}}(h)+\kappa_{j} k+\eta\left(\left(w_{1}\left(x^{*}\right)+\gamma\right)-h \inf _{x \in S_{i},|q|=1} \delta_{\epsilon}(x, q)+h \omega_{\delta_{\epsilon}}(h)+\kappa_{j} k\right)
$$

from which we conclude that

$$
\eta \leq \frac{h \omega_{\delta_{\epsilon}}(h)+\kappa_{j} k}{h \inf _{x \in S_{i},|q|=1} \delta_{\epsilon}(x, q)-h \omega_{\delta_{\epsilon}}(h)-\kappa_{j} k} .
$$

Estimating $\kappa_{j} \leq \beta(\varepsilon)^{-1} \inf _{x \in S_{j},|q|=1} \delta_{\epsilon}(x, q)+\omega_{\delta_{\epsilon}}(k)$ and writing $\mu_{j}=\inf _{x \in S_{j},|q|=1} \delta_{\epsilon}(x, q)$ (note that $\mu_{j} \geq \alpha(\epsilon)$ ) this becomes

$$
\eta \leq \frac{h \omega_{\delta_{\epsilon}}(h)+\beta(\varepsilon)^{-1} \mu_{j} k+\omega_{\delta_{\epsilon}}(k) k}{h \mu_{j}-h \omega_{\delta_{\epsilon}}(h)-\beta(\varepsilon)^{-1} \mu_{j} k-\omega_{\delta_{\epsilon}}(k) k} .
$$

Now we specify the assumption " $h, k>0$ sufficiently small" by choosing them such that $\omega_{\delta_{\epsilon}}(h)<<\alpha(\epsilon), k<<\beta(\varepsilon) h$ and $\omega_{\delta_{\epsilon}}(k) k<<\alpha(\epsilon) h$ which yields (recall $\alpha(\epsilon) \leq \mu_{j}$ )

$$
h \mu_{j}-h \omega_{\delta_{\epsilon}}(h)-\beta(\epsilon)^{-1} \mu_{j} k-\omega_{\delta_{\epsilon}}(k) k \geq \frac{1}{C} h \mu_{j}
$$


for some constant $C>0$ and thus

$$
\eta \leq C\left[\frac{\omega_{\delta_{\epsilon}}(h)}{\mu_{j}}+\frac{k}{\beta(\varepsilon) h}+\frac{\omega_{\delta_{\epsilon}}(k) k}{h \mu_{j}}\right]
$$

which implies the desired estimate for $w_{1}(x)+\gamma$ since $\mu_{j} \geq \alpha(\epsilon)$. Since all values in this resulting inequality are independent from $\gamma>0$ this also implies the estimate for $\gamma=0$.

The inequality for $u_{\epsilon}(x)-u_{\epsilon, h}^{k}(x)$ follows with the same technique and the obvious modifications using $w_{2}$; note that here the convexity of $\bar{\Omega}$ is also needed in Lemma 6.1 used in case (iii). Proceeding in this way we end up with the analogous estimate to (6.12)

$$
\eta \leq \frac{h \omega_{\delta_{\epsilon}}(h)+\kappa_{j} k}{h \inf _{x \in S_{i},|q|=1} \delta_{\epsilon}(x, q)}
$$

which leads to the desired result here without using the assumptions on $k$ and $h$.

\section{Proof of Proposition 5.6}

For any measurable and bounded $q$ and any $x \in \Omega$ denote the solution of (2.10) by $\xi(t, x, q(\cdot))$. Fix $\epsilon>0, \epsilon_{1}>0$ and $\epsilon_{2} \in(0, \epsilon)$ arbitrary and pick some $x \in \Omega$. Then by the optimal control representation of $U(2.10)-(2.11)$ there exists a solution $\xi_{\epsilon_{1}}(t)=\xi\left(t, x, q_{\epsilon_{1}}(\cdot)\right)$ with $\left|q_{\epsilon_{1}}(t)\right|=1$ and a time $T_{\epsilon_{1}}>0$ such that $\xi_{\epsilon_{1}}\left(T_{\epsilon_{1}}\right) \in \partial \Omega$ and

$$
\int_{0}^{T_{\epsilon_{1}}} \delta\left(\xi_{\epsilon_{1}}(t), q_{\epsilon_{1}}(t)\right) d t+g\left(\xi_{\epsilon_{1}}\left(T_{\epsilon_{1}}\right)\right)<U(x)+\epsilon_{1}
$$

We now divide the connected components $K_{\epsilon}^{i}, i \in I$ of $K_{\epsilon}$ into two classes by defining

$$
I_{1}:=\left\{i \in I \mid f(x) \geq \epsilon-\epsilon_{2} \text { for all } x \in K_{\epsilon}^{i}\right\}
$$

and $I_{2}=I \backslash I_{1}$. Then by the continuity of $H$ there exists a constant $\gamma\left(\epsilon_{2}\right)$ with $\gamma\left(\epsilon_{2}\right) \rightarrow 0$ as $\epsilon_{2} \rightarrow 0$ such that

$$
\left|\delta_{\epsilon}(x, p)-\delta(x, p)\right|<\gamma\left(\epsilon_{2}\right) \text { for all } x \in K_{\epsilon}^{i}, i \in I_{1},|p|=1 .
$$

Furthermore by the uniform continuity of $f$ every set $K_{\epsilon}^{i}, i \in I_{2}$, has a volume bounded from below by some uniform constant depending on $\epsilon_{2}$ and hence there are only finitely many of these sets; we may number them by $i=1, \ldots, N$.

Now we define for each of these $K_{\varepsilon}^{i}, i=1, \ldots, N$ which is hit by the trajectory $\xi_{\epsilon_{1}}$ times $t_{-}^{i}$ and $t_{+}^{i}$ by

$$
t_{-}^{i}=\inf \left\{t \in\left[0, T_{\epsilon_{1}}\right] \mid \xi_{\epsilon_{1}}(t) \in K_{\varepsilon}^{i}\right\} \text { and } t_{+}^{i}=\sup \left\{t \in\left[0, T_{\epsilon_{1}}\right] \mid \xi_{\epsilon_{1}}(t) \in K_{\varepsilon}^{i}\right\}
$$

where we omit those sets $K_{\varepsilon}^{i}$ for which $\left[t_{-}^{i}, t_{+}^{i}\right] \subset\left[t_{-}^{j}, t_{+}^{j}\right]$ for some $j \neq i$ holds. This gives us a finite number $r$ of pairwise disjoint intervals $\left[t_{-}^{i}, t_{+}^{i}\right]$ which we assume to be numbered according to their order, i.e. $t_{+}^{i}<t_{-}^{i+1}$ for $i=1, \ldots, r-1$.

For each trajectory piece $\left.\xi_{\epsilon_{1}}(\cdot)\right|_{\left[t_{+}^{i}, t_{-}^{i+1}\right]}$ we have by (6.13) and by the fact that outside $K_{\epsilon}$ the functions $\delta$ and $\delta_{\epsilon}$ coincide the estimate

$$
\int_{t_{+}^{i}}^{t_{-}^{i+1}}\left|\delta\left(\xi_{\epsilon_{1}}(t), q_{\epsilon_{1}}(t)\right)-\delta_{\epsilon}\left(\xi_{\epsilon_{1}}(t), q_{\epsilon_{1}}(t)\right)\right| d t \leq\left(t_{-}^{i+1}-t_{+}^{i}\right) \gamma\left(\epsilon_{2}\right) .
$$


For the points $\xi_{\epsilon_{1}}\left(t_{-}^{i}\right)$ and $\xi_{\epsilon_{1}}\left(t_{+}^{i}\right)$ we choose controls $q_{i}(\cdot)$ satisfying $\left|q_{i}(t)\right|=1$ and yielding $\xi\left(\tau_{i}, \xi_{\epsilon_{1}}\left(t_{-}^{i}\right), q_{i}(\cdot)\right)=\xi_{\epsilon_{1}}\left(t_{+}^{i}\right), \xi\left(\tau_{i}, \xi_{\epsilon_{1}}\left(t_{-}^{i}\right), q_{i}(\cdot)\right) \in K_{\epsilon}^{i}$ for all $t \in\left[0, \tau_{i}\right]$ and $\tau_{i} \leq d\left(K_{\epsilon}^{i}\right)$ which is possible by the definition of $d(\cdot)$ and the structure of the dynamics (2.10). We now define a sequence of times $t_{i}, i=0, \ldots, r$ by

$$
t_{0}=0, t_{1}=t_{-}^{1}, t_{i+1}=t_{i}+\tau_{i}+\left(t_{-}^{i+1}-t_{+}^{i}\right), i=1, \ldots, r-2, t_{r}=t_{r-1}+\tau_{r}+\left(T_{\epsilon_{1}}-t_{+}^{r}\right)
$$

and a measurable function $\tilde{q}(\cdot)$ by

$$
\tilde{q}(t)= \begin{cases}q_{\epsilon_{1}}(t), & t \in\left[t_{0}, t_{1}\right) \\ q_{i}\left(t-t_{i}\right), & t \in\left[t_{i}, t_{i}+\tau_{i}\right) \\ q_{\epsilon_{1}}\left(t-t_{i}-\tau_{i}+t_{+}^{i}\right), & t \in\left[t_{i}+\tau_{i}, t_{i+1}\right)\end{cases}
$$

This construction yields that

$$
\xi(t, x, \tilde{q}(\cdot))=\xi_{\epsilon_{1}}(t) \text { for all } t \in\left[t_{0}, t_{1}\right]
$$

and

$$
\xi(t, x, \tilde{q}(\cdot))=\xi_{\epsilon_{1}}\left(t-t_{i}-\tau_{i}+t_{+}^{i}\right) \text { for all } t \in\left[t_{i}+\tau_{i}, t_{i+1}\right], i=1, \ldots, r-1
$$

thus in particular it follows that $\xi\left(t_{r}, x, \tilde{q}(\cdot)\right)=\xi_{\epsilon_{1}}\left(T_{\epsilon_{1}}\right)$ and - defining $t_{+}^{0}:=0$ - we obtain

$$
\begin{aligned}
u_{\epsilon}(x)-g\left(\xi_{\epsilon_{1}}\left(T_{\epsilon_{1}}\right)\right) & \leq \int_{0}^{t_{r}} \delta_{\epsilon}(\xi(t, x, \tilde{q}(\cdot)), \tilde{q}(t)) d t \\
& =\sum_{i=0}^{r-1} \int_{t_{i}}^{t_{i+1}} \delta_{\epsilon}(\xi(t, x, \tilde{q}(\cdot)), \tilde{q}(t)) d t \\
& \leq \sum_{i=0}^{r-1}\left(\int_{t_{-}^{i}}^{t_{+}^{i+1}} \delta\left(\xi_{\epsilon_{1}}(t), q_{\epsilon_{1}}(t)\right) d t+\left(t_{-}^{i+1}-t_{+}^{i}\right) \gamma\left(\epsilon_{2}\right)+\tau_{i+1} c(\epsilon)\right) \\
& \leq U(x)-g\left(\xi_{\epsilon_{1}}\left(T_{\epsilon_{1}}\right)\right)+\epsilon_{1}+T_{\epsilon_{1}} \gamma\left(\epsilon_{2}\right)+d\left(K_{\epsilon}\right) c(\epsilon)
\end{aligned}
$$

Now letting first $\epsilon_{2} \rightarrow 0$ and then $\epsilon_{1} \rightarrow 0$ we obtain the assertion since $u_{\epsilon} \geq U$ is obvious.

\section{References}

[1] M. Bardi and I. Capuzzo Dolcetta, Optimal Control and Viscosity Solutions of Hamilton-Jacobi-Bellman equations, Birkhäuser, Boston, 1997.

[2] M. Bardi and M. Falcone, An approximation scheme for the minimum time function, SIAM J. Control Optim. 28 (1990), 950-965.

[3] G. Barles, and P. Souganidis, Convergence of approximation scheme for fully nonlinear second order equations, Asymptotic Anal. 4 (1991), 271-283.

[4] M.J.Brooks and B.K.P.Horn, Shape from Shading, The MIT Press, Boston, 1989. 
[5] A.M. Bruckstein and R.Kimmel, Global Shape from Shading, Comp. Vision and Image Understanding 62 (1991), 360-369.

[6] F. Camilli and A. Siconolfi, Maximal subsolutions for a class of degenerate HamiltonJacobi problems, Preprint 98/25, Dip. di Matematica, Univ. di Roma "La Sapienza", 1998.

[7] F.H.Clarke, Optimization and nonsmooth analysis, SIAM, Philadelphia, 1983.

[8] F. Colonius and W. Kliemann, Infinite fime optimal control and periodicity, Appl. Math. Optim. 20 (1989), 113-130.

[9] F. Colonius and W. Kliemann, Some aspects of control systems as dynamical systems, J. Dyn. Differ. Equ. 5 (1993), 469-494.

[10] L. Corrias, Fast Legendre-Fenchel transform and applications to Hamilton-Jacobi equations and conservation laws, SIAM J. Numer. Anal. 33 (1996), 1534-1558.

[11] M. Falcone, A numerical approach to the infinite horizon problem of deterministic control theory, Appl. Math. Optim. 15 (1987), 1-13. Corrigenda, ibid. 23 (1991), 213-214.

[12] M. Falcone and M. Sagona, An algorithm for the global solution of the Shape-fromShading model, in A. Del Bimbo, ed., "Image analysis and processing", 596-603, Lect. Notes in Computer Sciences 1310, Springer, 1997.

[13] L. Grüne, An adaptive grid scheme for the discrete Hamilton-Jacobi-Bellman equation, Numer. Math. 75 (1997), 319-337.

[14] H. Ishii and M. Ramaswamy, Uniqueness results for a class of Hamilton-Jacobi equations with singular coefficients, Comm. Par. Diff. Eq. 20 (1995), 2187-2213.

[15] P.L. Lions, Generalized solutions of Hamilton-Jacobi equations, Pitman, London, 1982.

[16] P.L. Lions, E. Rouy and A. Tourin, Shape-from-shading, viscosity solutions and edges, Numer. Math. 64 (1993), 323-353.

[17] E. Rouy and A. Tourin, A viscosity solution approach to Shape from shading, SIAM J. Num. Anal 29 (1992), 867-884. 